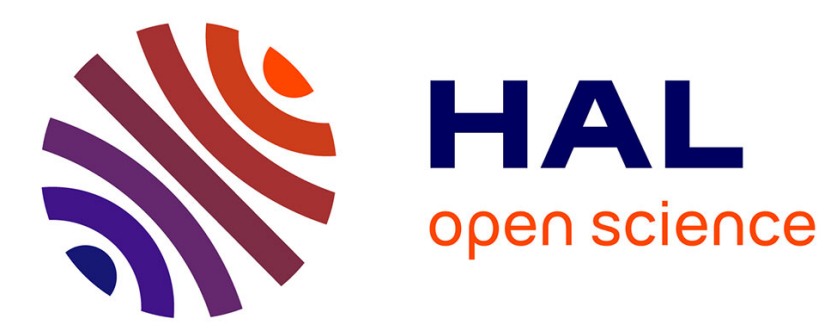

\title{
LMI Tests for Positive Definite Polynomials: Slack Variable Approach
}

\author{
Dimitri Peaucelle, Masayuki Sato
}

\section{To cite this version:}

Dimitri Peaucelle, Masayuki Sato. LMI Tests for Positive Definite Polynomials: Slack Variable Approach. IEEE Transactions on Automatic Control, 2009, 54 (4), pp.886-891. 10.1109/TAC.2008.2010971 . hal-02331395

\section{HAL Id: hal-02331395 https://hal.laas.fr/hal-02331395}

Submitted on 24 Oct 2019

HAL is a multi-disciplinary open access archive for the deposit and dissemination of scientific research documents, whether they are published or not. The documents may come from teaching and research institutions in France or abroad, or from public or private research centers.
L'archive ouverte pluridisciplinaire HAL, est destinée au dépôt et à la diffusion de documents scientifiques de niveau recherche, publiés ou non, émanant des établissements d'enseignement et de recherche français ou étrangers, des laboratoires publics ou privés. 


\title{
LMI Tests for Positive Definite Polynomials: Slack Variable Approach
}

\author{
Dimitri Peaucelle and Masayuki Sato
}

\begin{abstract}
The considered problem is assessing non-negativity of a function's values when indeterminates are in domains constrained by scalar polynomial inequalities. The tested functions are multi-indeterminates polynomial matrices which are required to be positive semi-definite. New tests based on linear matrix inequalities are provided in a Slack Variables type approach. The results are compared to those obtained via the Sum-OfSquares approach. They are proved to be equivalent in case of unbounded domains and less conservative if polytopic-type bounds are known.
\end{abstract}

Index Terms-LMI, Matrix-valued polynomials, Robustness, Positivity, Sum-Of-Squares

\section{INTRODUCTION}

About ten years after Shor has proposed the Sum-OfSquares (SOS) approach for obtaining the global minimum of polynomial functions [26], Chesi et al. [3], Lasserre [8] and Parrilo [14] have independently shown that SOS decomposition can be cast into semi-definite programming. After these first results, many publications taking advantage of SOS decomposition technique have been reported, e.g. nonlinear system analysis, hybrid system analysis, etc., some of which are surveyed in [13], [7]. But all positive polynomials are not SOS and Motzkin polynomials are examples of this fact. More precisely, as enunciated in Hilbert problems, the set of SOS polynomials coincides with the set of non-negative polynomials only in three very simple cases: single indeterminate (whatever degree), quadratic form (whatever number of indeterminates), and quartic form in two indeterminates [26], [18]. However, under some realistic restrictions, the gap between SOS polynomials and non-negative polynomials disappears. Lasserre has proved that all non-SOS polynomials, which are non-negative, can be approximated by other polynomials of higher degree which can be verified to be nonnegative with theory of moments [8], and they can also be approximated by some SOS polynomials with small SOS polynomial perturbations [9]. Further, polynomial functions, which are non-negative on unit ball, can be approximated by some SOS polynomials with small SOS polynomial perturbations [10]. Although most results are derived assuming scalar-valued polynomials, matrix-valued version of SOS approximation are also available. Proposed by Chesi et al. in [2] these are

D. Peaucelle is with LAAS-CNRS, Université de Toulouse, 7 avenue du Colonel Roche, 31077 Toulouse, FRANCE peaucelleelaas. fr

M. Sato is with Institute of Space Technology and Aeronautics, Japan Aerospace Exploration Agency, Mitaka, Tokyo 181-0015, Japan sato.masayuki@jaxa.jp exploited by Scherer and Hol in [25] with use of Putinar's SOS representation.

On the other hand, much research for robust stability/performance analysis has been conducted in the last decade using Slack Variable (SV) approach, e.g. [4], [28], [16], [6]. Based on Finsler lemma, the approach creates new variables that allow without much mathematical complications to derive LMI results with reduced conservatism. In addition to simplicity in matrix manipulations, the approach benefits of extensions for control design, [1], [15], [5]. But, the question whether conservatism of the SV approach can be further reduced remains an open problem. Partial answers have been provided. One (see [11], [12]) takes advantage of the fact that SV approach is in general applied to polynomials with indeterminates constrained in the unit simplex and results are obtained based on a matrix-valued version of Pólya's theorem [24]. Another (see [17], [20], [21]) uses redundant system modeling and may be interpreted as taking polynomial representations in basis of increasing degree. All these publications illustrate the influence of polynomial matrix inequalities representations for robustness analysis problems. However, to the authors' knowledge, no result has been reported for the relationship between SOS approach and SV approach. Based on preliminary results of [23], [22] this relationship is investigated in the present paper. SV approach is shown to encompass SOS approach.

The two upper cited techniques to be compared can be mathematically summarized as follows. For a given choice of a basis of monomials gathered in a vector $\breve{\theta}$ (in the present paper the elements of $\breve{\theta}$ are of the form $\prod_{k} \theta_{k}^{m_{k}}$ where the $\theta_{k}$ are the scalar indeterminates), a matrix valued polynomial has at least one representation such that $F(\theta)=(\breve{\theta} \otimes \mathbf{1})^{T} \hat{F}(\breve{\theta} \otimes \mathbf{1})$, where $\mathbf{1}$ stands for the identity matrix of the dimension of $F(\theta)$ and $\otimes$ is the Kronecker product. The elements of the $\hat{F}$ matrix are the coefficient matrices of the polynomial. SOS result states that $F(\theta)$ is positive semi-definite for all indeterminates $\theta$ if there exists a matrix $\hat{L}$ such that $(\breve{\theta} \otimes \mathbf{1})^{T} \hat{L}(\breve{\theta} \otimes \mathbf{1})=\mathbf{0}$ and $\hat{F}+\hat{L}$ is positive semi-definite. $F(\theta)$ has in such case a factorization as a SOS. SV technique is based on Finsler lemma and on the fact that there exists an affine with respect to $\theta$ matrix $\Psi(\theta)$ such that $\Psi^{T}(\theta)(\breve{\theta} \otimes \mathbf{1})=\mathbf{0}$. SV result states that $F(\theta)$ is positive semi-definite for $\theta$ in the convex set of a finite number of vertices $\theta^{[p]}$ if there exists a matrix $N$ such that $\hat{F}+\Psi\left(\theta^{[p]}\right) N+N^{T} \Psi^{T}\left(\theta^{[p]}\right)$ is positive semi-definite for all vertices. In the paper we extend this last SV result for the case when indeterminates are unbounded (not in a polytope) and prove the direct relation between the $N$ and $\hat{L}$ matrices. 
The outline is as follows. The next section is devoted to (inevitably intricate) notations and to the problem definition: proving a polynomial matrix values are positive semi-definite for indeterminates constrained in the intersection of polytopes and scalar polynomial inequalities. Section III states the main results, namely the LMI formulas obtained via the SV approach, and shows equivalence of SV and SOS methods in case of unbounded polytopic constraints. The byproducts of the SV approach is a less conservative result in case of bounded indeterminate domains and an easy to code reformulation of SOS results. Section IV considers the mixed bounded/unbouded case and formulates results for solving robust polynomial semi-definite programs. A conclusion ends the paper.

\section{PREliminaries}

\section{General notations}

$\mathrm{R}^{n \times m}, \mathrm{~S}^{n}$ and $\mathrm{Q}^{n}$, denote respectively the sets of $n \times m$ real, symmetric $n \times n$ real and skew-symmetric $n \times n$ real matrices. For two symmetric matrices, $A$ and $B, A>(\geq) B$ means that $A-B$ is positive (semi-) definite. $A^{T}$ denotes the transpose of $A . \mathbf{1}_{n}, \mathbf{0}_{m, n}$ and $\mathbf{0}_{n}$ denote respectively the identity matrix of size $n$, null matrix of size $m \times n$ and the square null matrix of size $n \times n$. If the context allows it, the dimensions of these matrices are sometimes omitted. For a given matrix $B \in \mathrm{R}^{m \times n}$ such that $\operatorname{rank}(B)=r$, define $B^{\perp} \in \mathrm{R}^{n \times(n-r)}$ the right orthogonal complement of $B$ such that $B B^{\perp}=\mathbf{0}$ and $B^{\perp} B^{\perp T}>\mathbf{0}$. The notation $\langle A\rangle$ stands for the symmetric matrix $A+A^{T}$. Trivially $\langle A\rangle=\mathbf{0}$ if and only if $A$ is skew-symmetric and $\langle A\rangle=2 A$ if and only if $A$ is symmetric. $\otimes$ stands for the Kronecker product (matrix direct product). For $A=\left[a_{i j}\right] \in \mathrm{R}^{n \times m}$ and $B \in \mathrm{R}^{p \times q}$ it is such that $C=A \otimes B$ is a $\mathrm{R}^{n p \times m q}$ matrix with block entries $C=\left[a_{i j} B\right]$. For matrices of appropriate dimensions, the Kronecker product is such that $(A \otimes B)(C \otimes D)=(A C) \otimes(B D)$.

\section{Problem definition}

Consider $\bar{k}$ real scalar independent indeterminates $\theta_{1}, \ldots \theta_{\bar{k}}$ and define $\theta=\left(\begin{array}{lll}\theta_{1} & \ldots & \theta_{\bar{k}}\end{array}\right)^{T}$ the vector of all indeterminates. Consider $F$ a symmetric-matrix valued polynomial function of the indeterminates, $F(\theta) \in \mathrm{S}^{n}$. The goal of the paper is to give LMI tests to prove $F(\theta)$ is positive semidefinite for $\theta$ constrained in the intersection of two type of sets

$$
F(\theta) \geq \mathbf{0}_{n} \quad, \quad \forall \theta \in \mathcal{G} \cap \mathcal{P}
$$

defined as follows.

- Consider $\bar{q}$ scalar polynomials of the indeterminates $\theta_{1}, \ldots \theta_{\bar{k}}, g_{q}(\theta) \in \mathrm{R}, q=1 \ldots \bar{q}$. The first type of set is such that these polynomials are non-negative:

$$
\mathcal{G}=\left\{\theta \in \mathrm{R}^{\bar{k}} \quad: \quad g_{q}(\theta) \geq 0, q=1 \ldots \bar{q}\right\} .
$$

Such set is possibly unbounded and may be nonconnected.

- Consider $\mathcal{K}_{b} \subset\{1 \ldots \bar{k}\}$ be a set of indeterminates' indexes and let $\bar{k}_{b}$ be the number of elements in the set. Define $\vartheta \in \mathrm{R}^{\bar{k}_{b}}$ the vector of indeterminates $\theta_{k \in \mathcal{K}_{b}}$ ordered with growing index. The second type of set is a semi-polytope defined as the intersection of $\mathrm{R}^{\bar{k}}$ with a polytope of the indeterminates $\vartheta$ having $\bar{p}$ vertices $\vartheta^{[p]}$ :

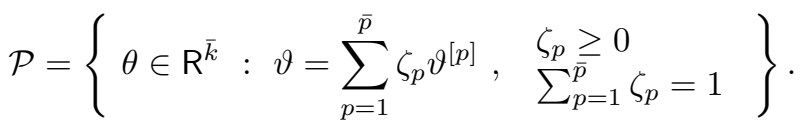

In such set, the indeterminates $\theta_{k \in \mathcal{K}_{b}}$ are bounded.

Two special and important cases are studied in the next section: first, the (fully) bounded case when $\bar{k}_{b}=\bar{k}$; second, the unbounded case when $\bar{k}_{b}=0$. Based on these results, section IV gives extensions to the mixed bounded/unbounded case $\left(0<\bar{k}_{b}<\bar{k}\right)$.

\section{Notations for polynomials manipulations}

For each inderterminate $\theta_{k}$ define $\breve{\theta}_{k}^{m_{k}}=$ $\left(\begin{array}{llll}1 & \theta_{k} & \ldots & \theta_{k}^{m_{k}}\end{array}\right)^{T} \in \mathrm{R}^{m_{k}+1}$ the vector of powers up to power $m_{k}$. The notation $\breve{\theta}=\breve{\theta}_{1}^{m_{1}} \otimes \breve{\theta}_{2}^{m_{2}} \ldots \otimes \breve{\theta}_{\bar{k}}^{m_{\bar{k}}}$ produces a vector of all monomials obtained as products of elements $\theta_{k}^{j}$ with indeterminates indices $k$ ranging from 1 to $\bar{k}$ and powers going up to $m_{k}$. In the following, the polynomials are expressed in this basis of monomials. Note that this basis is very general and maybe over-dimensioned for given polynomials and lead to increased computational effort. To handle this issue one may consider Groebner bases methods. But for the purpose of comparison between SV and SOS the general basis is needed and the use of Groebner bases is left for future work.

For the sake of readability of formulas define the notation $\sigma_{k_{1}, k_{2}}=\prod_{k=k_{1}}^{k_{2}}\left(m_{k}+1\right)$ for all $1 \leq k_{1} \leq k_{2} \leq \bar{k}$ and let $\sigma_{1,0}=1$ and $\sigma_{\bar{k}+1, \bar{k}}=1$. Moreover, let $\sigma=\sigma_{1, \bar{k}}$ and define $\pi_{k}=\sigma m_{k} /\left(m_{k}+1\right)$ for all $k \in\{1 \ldots \bar{k}\}$.

For each $k \in\{1 \ldots \bar{k}\}$ define $\bar{m}_{k}$ as the maximal degree of the entries of the matrix polynomial $F$ and of the $g_{q}$ polynomials with respect to the indeterminate $\theta_{k}$. For any sequence $\left\{m_{k}\right\}_{k=1 \ldots \bar{k}}$ such that $2 m_{k} \geq \bar{m}_{k}$, the $F$ and $g_{q}$ polynomials can be expressed as

$$
F(\theta)=\left(\breve{\theta}^{T} \otimes \mathbf{1}_{n}\right) \hat{F}\left(\breve{\theta} \otimes \mathbf{1}_{n}\right) \quad, \quad g_{q}(\theta)=\breve{\theta}^{T} \hat{G}_{q} \breve{\theta}
$$

where $\hat{F} \in \mathrm{S}^{n \sigma}$ and $\hat{G}_{q} \in \mathrm{S}^{\sigma}$. Note that the coefficients matrices $\hat{F}$ and $\hat{G}_{q}$ are non unique, especially if the maximal degrees $m_{k}$ are chosen large. Given the sequence $\left\{m_{k}\right\}_{k=1 \ldots \bar{k}}$, construction of an admissible choice of $\hat{F}$ and $\hat{G}_{q}$ matrices may be tedious but does not make any problem. $\hat{F}$ and $\hat{G}_{q}$ are further assumed to be given.

Slack Variable approach is based on the use of elimination lemma [27] (a variation on Finsler lemma) that states inequality $B^{\perp T} Q B^{\perp}>\mathbf{0}$ is equivalent to the existence of a matrix $N$ such that $Q+\langle N B\rangle>\mathbf{0}$. The key idea for producing LMI results is that "creating" the additional variable $N$ may transform a polynomial inequality $\left(B(\theta)^{\perp}\right.$ is a polynomial with respect to indeterminates $\theta$ ) into a linear inequality $(B(\theta)$ is affine with respect to $\theta)$. This linearization is usually achieved at the expense of some conservatism because $N$ is chosen independent of $\theta$. Yet, in the present paper the inherent 
conservatism of the method is reduced thanks to the use of multiple slack variables, one per indeterminate. Notations for this multiple slack variables creation are as follows.

For each $k \in\{1 \ldots \bar{k}\}$, define the following two constant matrices $\eta_{k}^{[0]}=\left[\begin{array}{c}\mathbf{0}_{1, m_{k}} \\ -\mathbf{1}_{m_{k}}\end{array}\right], \eta_{k}^{[\infty]}=\left[\begin{array}{c}\mathbf{1}_{m_{k}} \\ \mathbf{0}_{1, m_{k}}\end{array}\right]$. The vector $\breve{\theta}_{k}^{m_{k}}$ of power series of $\theta_{k}$ happens to be a right orthogonal complement of an affine expression of these two matrices: $\breve{\theta}_{k}^{m_{k}}=\left(\left(\eta_{k}^{[0]}+\theta_{k} \eta_{k}^{[\infty]}\right)^{T}\right)^{\perp}$. Moreover one gets that the following equation on the vector of all monomials $\breve{\theta}$

$$
\left(\breve{\theta}^{T} \otimes \mathbf{1}_{n}\right)\left(\mathbf{1}_{\sigma_{1, k-1}} \otimes\left(\eta_{k}^{[0]}+\theta_{k} \eta_{k}^{[\infty]}\right) \otimes \mathbf{1}_{n \sigma_{k+1, \bar{k}}}\right)=\mathbf{0}
$$

holds whatever $k=1 \ldots \bar{k}$ and whatever $\theta \in \mathrm{R}^{\bar{k}}$ (see equation (11) given in Appendix for details about this fact). Rather than considering $\breve{\theta}$ as a right orthogonal complement for a matrix dependent of all indeterminates $\theta_{1} \ldots \theta_{\bar{k}}$, the upper formula is used in the paper to create separate slack variables with respect to each indeterminate.

For compactness of formulas define the following matrices

$$
\begin{aligned}
& \Psi_{k}^{[0]}=\left(\mathbf{1}_{\sigma_{1, k-1}} \otimes \eta_{k}^{[0]} \otimes \mathbf{1}_{n \sigma_{k+1, \bar{k}}}\right) \\
& \Psi_{k}^{[\infty]}=\left(\mathbf{1}_{\sigma_{1, k-1}} \otimes \eta_{k}^{[\infty]} \otimes \mathbf{1}_{n \sigma_{k+1, \bar{k}}}\right)
\end{aligned}
$$

and $\Psi_{k}\left(\theta_{k}\right)=\Psi_{k}^{[0]}+\theta_{k} \Psi_{k}^{[\infty]}$. With this notations, formula (5) read as $\left(\breve{\theta}^{T} \otimes \mathbf{1}_{n}\right) \Psi_{k}\left(\theta_{k}\right)=\mathbf{0}$. Moreover, in case $k \in \mathcal{K}_{b}$, define $\Psi_{k}^{[p]}=\Psi_{k}\left(\theta_{k}^{[p]}\right)$ which is such that $\Psi_{k}\left(\theta_{k}\right)=\sum_{p=1}^{\bar{p}} \zeta_{p} \Psi_{k}^{[p]}$ (according to notations in (3)).

\section{Slack Variables Results}

The first result is for the case of bounded indeterminates in a polytope. This case corresponds to the usual framework in which SV approach has been developed [4], [16], [6]. At the difference of these references, the Theorem generates several slack variables $\left(N_{k}\right)$, rather than just one. This modification, that increases the numerical complexity, provides new degrees of freedom useful in the following to prove new properties.

Theorem 1: Assume $\bar{k}_{b}=\bar{k}$, all indeterminates are bounded inside a given polytope. Property (1) holds if there exist $\bar{q} \bar{p}$ symmetric matrices $H_{q}^{[p]} \in \mathrm{S}^{n}$ and $\bar{k}$ matrices $N_{k} \in \mathrm{R}^{n \pi_{k} \times n \sigma}$ such that for all $(p, q) \in\{1 \ldots \bar{p}\} \times\{1 \ldots \bar{q}\}$ the following LMI conditions are feasible: $H_{q}^{[p]} \geq \mathbf{0}_{n}$ and

$$
\hat{F}+\left\langle\sum_{k=1}^{\bar{k}} \Psi_{k}^{[p]} N_{k}\right\rangle \geq \sum_{q=1}^{\bar{q}} \hat{G}_{q} \otimes H_{q}^{[p]} .
$$

Proof: Define $\theta \in \mathcal{P}$ any element of the polytope parameterized by the $\bar{p}$ scalars $\zeta_{p}$ as in (3). Let $H_{q}(\theta)=$ $\sum_{p=1}^{\bar{p}} \zeta_{p} H_{q}^{[p]}$. The inequalities (6) are linear with respect to the vertices $\theta^{[p]}$ and $H_{q}^{[p]}$ therefore by convexity they hold as well for all values $\theta$ in the polytope $\mathcal{P}: H_{q}(\theta) \geq \mathbf{0}_{n}$ and

$$
\hat{F}+\left\langle\sum_{k=1}^{\bar{k}} \Psi_{k}\left(\theta_{k}\right) N_{k}\right\rangle \geq \sum_{q=1}^{\bar{q}} \hat{G}_{q} \otimes H_{q}(\theta) .
$$

Pre and post-multiply inequalities (7) by $\breve{\theta}^{T} \otimes \mathbf{1}_{n}$ and its transpose respectively. One gets due to formulas (5):

$$
\forall \theta \in \mathcal{P} \quad: \quad H_{q}(\theta) \geq \mathbf{0}_{n} \quad, \quad F(\theta) \geq \sum_{q=1}^{\bar{q}} g_{q}(\theta) H_{q}(\theta) .
$$

Hence $F(\theta) \geq \mathbf{0}_{n}$ for all $\theta \in \mathcal{G} \cap \mathcal{P}$.

The unbounded case is now considered. It is to our knowledge the first time such SV result is produced without constraining indeterminates inside polytopes. As suggested by the notations it can be interpreted as taking vertices at infinity.

Theorem 2: Assume $\bar{k}_{b}=0$, no bound is a priori known on the indeterminates. Property (1) holds if there exist $\bar{q}$ symmetric matrices $H_{q} \in \mathrm{S}^{n}$ and $\bar{k}$ skew-symmetric matrices $\hat{N}_{k} \in \mathrm{Q}^{n \pi_{k}}$ such that for all $q \in\{1 \ldots \bar{q}\}$ the following LMI conditions are feasible:

$$
H_{q} \geq \mathbf{0}_{n}, \hat{F}+\left\langle\sum_{k=1}^{\bar{k}} \Psi_{k}^{[0]} \hat{N}_{k} \Psi_{k}^{[\infty] T}\right\rangle \geq \sum_{q=1}^{\bar{q}} \hat{G}_{q} \otimes H_{q} .
$$

Proof: The $\hat{N}_{k}$ matrices being skew-symmetric one obtains that $\left\langle\left(\Psi_{k}\left(\theta_{k}\right) \hat{N}_{k} \Psi_{k}^{[\infty] T}\right\rangle=\left\langle\Psi_{k}^{[0]} \hat{N}_{k} \Psi_{k}^{[\infty] T}\right\rangle\right.$. The matrices $N_{k}=\hat{N}_{k} \Psi_{k}^{[\infty] T}$ and $H_{q}(\theta)=H_{q}$ therefore satisfy conditions (7) for all $\theta \in \mathrm{R}^{\bar{k}}$. The remaining follows the lines of Theorem's 1 proof.

One major issue of the paper is to give a comparison between SV and SOS approaches. For the unbounded case the two happen to be exactly the same as stated by the next Theorem. Therefore it implies that all results known in the SOS context may be generalized to the SV approach. In particular, when $\mathcal{G} \cap \mathcal{P}=\mathrm{R}^{\bar{k}}$ is unconstrained, exactness of the LMI tests is guaranteed for the following cases: $\bar{k}=1$ (single indeterminate polynomials); $m_{k} \leq 1$ (quadratic forms); $\bar{k}=2$ and $m_{k} \leq 2$ (quartic forms of two indeterminates). Moreover, asymptotic convergence of LMI conditions to exact tests for problem (1) as the degree of the basis of monomials grows (under particular assumptions on the constraints, see [25]) keeps true for the SV approach. More precisely, to obtain the exact equivalence of the SV approach with results for the SOS approach in [25], it is needed to extend the results to $H_{q}$ defined as SOS polynomial matrices. This may be done without any theoretical obstacle but needs involved mathematical manipulations (see for example papers [22], [19]). We have limited our study to the case of zero degree $H_{q}$ to limit this mathematical manipulation complexity.

Theorem 3: The LMI conditions (8) hold if and only if the matrix-valued polynomial $F(\theta)-\sum_{q=1}^{\bar{q}} g_{q}(\theta) H_{q}$ is SOS.

To prove the Theorem, recall (see [25]) that a polynomial $S(\theta)=\left(\breve{\theta}^{T} \otimes \mathbf{1}_{n}\right) \hat{S}\left(\breve{\theta} \otimes \mathbf{1}_{n}\right)$ is SOS if and only if $\hat{S}+\hat{L}$ is positive semi-definite for some matrix $\hat{L}$ constrained such that $\left(\breve{\theta}^{T} \otimes \mathbf{1}_{n}\right) \hat{L}\left(\breve{\theta} \otimes \mathbf{1}_{n}\right)$ is identically zero for all $\theta \in \mathbf{R}^{\bar{k}}$. Theorem 3 therefore hold if all such matrices $\hat{L}$ have a multiple Slack Variables formulation: $\hat{L}=\left\langle\sum_{k=1}^{\bar{k}} \Psi_{k}^{[0]} \hat{N}_{k} \Psi_{k}^{[\infty] T}\right\rangle$ for some skew-symmetric matrices $\hat{N}_{k}$. This corresponds to the following lemma (when $\alpha=1$ and $\beta=n$ ). 
Lemma 1: Let $\alpha$ and $\beta$ be two integers. The multiindeterminate matrix polynomial $L(\theta)=\left(\mathbf{1}_{\alpha} \otimes \breve{\theta}^{T} \otimes\right.$ $\left.\mathbf{1}_{\beta}\right) \hat{L}\left(\mathbf{1}_{\alpha} \otimes \breve{\theta} \otimes \mathbf{1}_{\beta}\right)$ is identically zero for all $\theta \in \mathrm{R}^{\bar{k}}$ if and only if there exists $\bar{k}$ skew-symmetric matrices $\hat{N}_{k}$ such that

$$
\begin{array}{r}
\hat{L}=\left\langle\sum_{k=1}^{\bar{k}}\left(\mathbf{1}_{\alpha \sigma_{1, k-1}} \otimes \eta_{k}^{[0]} \otimes \mathbf{1}_{\beta \sigma_{k+1, \bar{k}}}\right) \hat{N}_{k} \ldots\right. \\
\left.\left(\mathbf{1}_{\alpha \sigma_{1, k-1}} \otimes \eta_{k}^{[\infty] T} \otimes \mathbf{1}_{\beta \sigma_{k+1, \bar{k}}}\right)\right\rangle .
\end{array}
$$

Proof: The 'if' part is similar to the proof of Theorem 2 and is therefore omitted. The 'only if' part of the proof needs many more derivations and is therefore given in appendix at the end of the paper.

Lemma 1 can also be seen as a simple to code formula for generating the matrix $\hat{L}$ of the SOS approach. Unfortunately the drawback is that the number of decision variables contained in the $\hat{N}_{k}$ matrices is larger than those needed in SOS. It is thus a simple to code but over-parameterized formula. To reduce the numerical complexity it is also possible to structure some more the $\hat{N}_{k}$ matrices following the ideas in [23], [22]. The reason for these structures can be found in the details of the proof given in appendix.

\section{A. Mixed bounded/unbounded case}

Theorem 4: Property (1) holds if there exist $\bar{q} \bar{p}$ symmetric matrices $H_{q}^{[p]} \in \mathrm{S}^{n}, \bar{k}_{b}$ matrices $N_{k} \in \mathrm{R}^{n \pi_{k} \times n \sigma}$ without any structure and $\left(\bar{k}-\bar{k}_{b}\right)$ skew-symmetric matrices $\hat{N}_{k} \in \mathrm{Q}^{n \pi_{k}}$, such that for all $(p, q) \in\{1 \ldots \bar{p}\} \times\{1 \ldots \bar{q}\}$ the following LMI conditions are feasible: $H_{q}^{[p]} \geq \mathbf{0}_{n}$ and

$\hat{F}+\left\langle\sum_{k \notin \mathcal{K}_{b}} \Psi_{k}^{[0]} \hat{N}_{k} \Psi_{k}^{[\infty] T}+\sum_{k \in \mathcal{K}_{b}} \Psi_{k}^{[p]} N_{k}\right\rangle \geq \sum_{q=1}^{\bar{q}} \hat{G}_{q} \otimes H_{q}^{[p]}$.

Theorem 4 is a trivial combination of both previous result. It allows to reduce conservatism of the unbounded case if some of the indeterminates are known to be constrained in bounded sets as stated in the following corollary.

Corollary 1: If the set $\mathcal{G}$ is known to be bounded or partially bounded with respect to some indeterminates $\theta_{k \in \mathcal{K}_{b}}$, then, let any over-bounding semi-polytopic set, $\mathcal{P} \supset \mathcal{G}$, the LMIs (10) are feasible if (8) is feasible.

This corollary indicates that if the constraints $\mathcal{G}$ define a bounded (or partially bounded) region, then the SV result is less conservative than the SOS result (assuming identical representations $\hat{F}, \hat{G}_{q}$ of the polynomials). As said previously (just before Theorem 3) this comparison is here done for the case when one restricts the variables $H_{q}$ to be indeterminate independent. These can also be chosen as polynomials (of degree such that $g_{q}(\theta) H_{q}(\theta)$ are of degrees $2 m_{k}$ with respect to each indeterminate $\theta_{k}$ ) in which case the comparison between SV and SOS holds as well for the same reasons. Still, conservatism reduction provided by the slack variables for bounded indeterminates is at the expense of increasing drastically the number of variables and the size of the LMIs (by a factor $\bar{p}$ ). Not to make this factor too large, one may consider cross-polytopes with $\bar{p}=2 \bar{k}$ vertices defined as

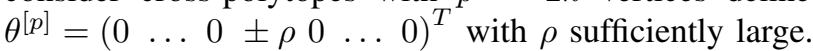

Numerical examples with comparisons of the SV and SOS methods can be found in [23], [22]. They illustrate both the efficiency of the contribution and its drawbacks in terms of numerical complexity. For space reasons these numerical examples are not reproduced in the present paper.

\section{CONCLUSIONS}

Aiming at the origin to compare Slack Variables (SV) and Sum-Of-Squares (SOS) approaches, we finally obtain in this paper both, an easy to code formulation of SOS results, and a generalization of the SV approach for non-bounded indeterminates. SV results moreover prove to be less conservative than SOS in case of bounded indeterminates, yet more demanding in terms of numerical burden. As attested by the numerous papers adopting SV approach (sometimes implicitly without direct references to the method), this technique is quite popular for robustness analysis in case of polytopic uncertainties but up to now seemed not to have extensions for other types of uncertainties. The exposed results allow these extensions where, as suggested by the notations, vertices of the polytope are replaced by matrices describing the parameters at the origin and at infinity.

Acknowledgments: The authors gratefully acknowledge Professor Shinji Hara as well as Didier Henrion for fruitful discussions and helpful comments. They also thank the anonymous reviewers for their attentive reading and their valuable remarks.

\section{REFERENCES}

[1] P. Apkarian, P.C. Pellanda, and H.D. Tuan. Mixed $H_{2} / H_{\infty}$ multichannel linear parameter-varying control in discrete time. In American Control Conference, pages 1322-1326, Chicago, Il, June 2000.

[2] G. Chesi, A. Garulli, A. Tesi, and A. Vicino. Polynomially parameterdependent Lyapunov functions for robust stability of polytopic systems: An LMI approach. IEEE Trans. on Automat. Control, 50(3):365-370, March 2005.

[3] G. Chesi, A. Tesi, A. Vicino, and R. Genesio. On convexification of some minimum distance problems. In European Control Conference, Karlsruhe, 1999.

[4] M.C. de Oliveira, J. Bernussou, and J.C. Geromel. A new discretetime stability condition. Systems \& Control Letters, 37(4):261-265, July 1999.

[5] Y. Ebihara and T. Hagiwara. New dilated LMI characterizations for continuous-time multiobjective controller synthesis. Automatica, 40(11):2003-2009, 2004.

[6] Y. Ebihara and T. Hagiwara. A dilated LMI approach to robust performance analysis of linear time-invariant uncertain systems. Automatica, 41(11):1933-1941, 2005.

[7] D. Henrion and A. Garulli, editors. Positive Polynomials in Control. Lecture Notes in Control and Information Sciences 312, Springer Verlag, Berlin, 2005.

[8] J.B. Lasserre. Global optimization with polynomials and the problem of moments. SIAM J. on Optimization, 11(3):796-817, 2001.

[9] J.B. Lasserre. A sum of squares approximation of nonnegative polynomials. SIAM J. on Optimization, 16(3):751-765, 2006.

[10] J.B. Lasserre and T. Netzer. SOS approximation of nonnegative polynomials via simple high degree perturbations. Math. Zeitschrift, 256:99-112, 2007. 
[11] R.C.L.F. Oliveira and P.L.D Peres. Stability of polytopes of matrices via affine parameter-dependent Lyapunov functions: Asymptotically exact LMI conditions. Linear Algebra and Its Applications, 405:209-228, 2005.

[12] R.C.L.F. Oliveira and P.L.D. Peres. LMI conditions for robust stability analysis based on polynomially parameter-dependent Lyapunov functions. Systems \& Control Letters, 55:52-61, 2006.

[13] A. Papachristodoulou and S. Prajna. A tutorial on sum of squares techniques for system analysis. In American Control Conference, pages 2686-2700, Portland, June 2005.

[14] P.A. Parillo. Semidefinite programming relaxations for semialgebraic problems. Mathematical Programing, 96(2):293-320, 2003.

[15] D. Peaucelle and D. Arzelier. An efficient numerical solution for $\mathrm{H}_{2}$ static output feedback synthesis. In European Control Conference, pages 3800-3805, Porto, Portugal, September 2001.

[16] D. Peaucelle, D. Arzelier, O. Bachelier, and J. Bernussou. A new robust D-stability condition for real convex polytopic uncertainty. Systems \& Control Letters, 40(1):21-30, May 2000.

[17] D. Peaucelle, Y. Ebihara, D. Arzelier, and T. Hagiwara. General polynomial parameter-dependent Lyapunov functions for polytopic uncertain systems. In International Symposium on Mathematical Theory of Networks and Systems, Kyoto, July 2006.

[18] Reznick. "Some Concrete Aspects of Hilbert's 17th Problem," in contemporary mathematics. American Mathematical Society, 253:251272, 2000

[19] M. Sato. Robust stability/performance analysis for polytopic systems via multiple slack variable approach. In IFAC World Congress, pages 11391-11396, Seoul, July 2008.

[20] M. Sato and D. Peaucelle. Robust stability/performance analysis for linear time invariant polynomially parameter-dependent systems using polynomially parameter-dependent Lyapunov functions. In IEEE Conference on Decision and Control, San Diego, December 2006.

[21] M. Sato and D. Peaucelle. Robust stability/performance analysis for linear time invariant polytopically parameter-dependent systems using polynomially parameter-dependent Lyapunov functions. In IEEE Conference on Decision and Control, San Diego, December 2006.

[22] M. Sato and D. Peaucelle. Comparison between SOS approach and slack variable approach for non-negativity check of polynomial functions: multiple variable case. In European Control Conference, Kos, July 2007.

[23] M. Sato and D. Peaucelle. Comparison between SOS approach and slack variable approach for non-negativity check of polynomial functions: single variable case. In American Control Conference, New-York, July 2007.

[24] C.W. Scherer. Relatations for robust linear matrix inequality problems with verifications for exactness. SIAM J. Matrix Anal. Appl., 27(2):365$395,2005$.

[25] C.W. Scherer and C.W.J. Hol. Matrix sum-of-squares relaxations for robust semi-definite programs. Mathematical Programing, 107(12):189-211, 2006

[26] N.Z. Shor. Class of global minimum bounds of polynomial functions. Cybernetics, 23(6):731-734, 1987.

[27] R.E. Skelton, T. Iwasaki, and K. Grigoriadis. A Unified Approach to Linear Control Design. Taylor and Francis series in Systems and Control, 1998.

[28] A. Trofino Neto. Parameter dependent Lyapunov functions for a class of uncertain linear systems: an LMI approach. In IEEE Conference on Decision and Control, pages 2341-2346, Phoenix, December 1999.

\section{APPENDIX}

This appendix is dedicated to the proof of Lemma 1. For this end notations and preliminary technical results are exposed.

Define $\theta_{\left[k_{1}, k_{2}\right]}=\left(\begin{array}{lll}\theta_{k_{1}} & \ldots & \theta_{k_{2}}\end{array}\right)^{T}$ the vector of indeterminates with indices $k$ ranging from $k_{1}$ to $k_{2}$. As previously $\breve{\theta}_{k}^{m_{k}}=\left(\begin{array}{llll}1 & \theta_{k} & \ldots & \theta_{k}^{m_{k}}\end{array}\right)^{T} \in \mathrm{R}^{m_{k}+1}$ is the vector of powers of each indeterminate $\theta_{k}$ up to power $m_{k}$. Let $\breve{\theta}_{\left[k_{1}, k_{2}\right]}=\breve{\theta}_{k_{1}}^{m_{k_{1}}} \otimes \breve{\theta}_{k_{1}+1}^{m_{k_{1}+1}} \ldots \otimes \breve{\theta}_{k_{2}}^{m_{k_{2}}}$ be the vectors of all monomials obtained as products of elements $\theta_{k}^{j}$ with indeterminates indices $k$ ranging from $k_{1}$ to $k_{2}$ and powers going up to $m_{k}$. The vectors $\mathscr{\theta}_{\left[k_{1}, k_{2}\right]}$ have exactly $\sigma_{k_{1}, k_{2}}=\prod_{k=k_{1}}^{k_{2}}\left(m_{k}+1\right)$ elements. Recall that by definition $\sigma_{1,0}=1$ and $\sigma_{\bar{k}+1, \bar{k}}=1$.
Recall as well the notation $\pi_{k}=\sigma m_{k} /\left(m_{k}+1\right)$. For compactness of formulas, $\theta=\theta_{[1, \bar{k}]}, \breve{\theta}=\breve{\theta}_{[1, \bar{k}]}$ and $\sigma=\sigma_{1, \bar{k}}$. The notations are such that whatever $k \in\{1 \ldots \bar{k}\}, \breve{\theta}=$

$$
\begin{aligned}
& =\breve{\theta}_{[1, k-1]} \otimes \breve{\theta}_{k}^{m_{k}} \otimes \breve{\theta}_{[k+1, \bar{k}]} \\
& =\left(\breve{\theta}_{[1, k-1]} \otimes \mathbf{1}_{\sigma_{k, k}} \otimes \breve{\theta}_{[k+1, \bar{k}]}\right)\left(1 \otimes \breve{\theta}_{k}^{m_{k}} \otimes 1\right) \\
& =\left(\mathbf{1}_{\sigma_{1, k-1}} \otimes \breve{\theta}_{k}^{m_{k}} \otimes \mathbf{1}_{\sigma_{k+1, \bar{k}}}\right)\left(\breve{\theta}_{[1, k-1]} \otimes 1 \otimes \breve{\theta}_{[k+1, \bar{k}]}\right) .
\end{aligned}
$$

This type of factorization of one among all indeterminates is of major importance for deriving the results.

Lemma 2: Let $\alpha$ and $\beta$ two integers and assume $\hat{L}(\theta)$ is a matrix polynomial of several scalar indeterminates. If the following two conditions hold

$$
\begin{gathered}
\left(\mathbf{1}_{\alpha} \otimes e_{1}^{T} \otimes \mathbf{1}_{\beta}\right) \hat{L}(\theta)\left(\mathbf{1}_{\alpha} \otimes e_{1} \otimes \mathbf{1}_{\beta}\right)=\mathbf{0} \\
\left(\mathbf{1}_{\alpha} \otimes e_{l+1}^{T} \otimes \mathbf{1}_{\beta}\right) \hat{L}(\theta)\left(\mathbf{1}_{\alpha} \otimes e_{l+1} \otimes \mathbf{1}_{\beta}\right)=\mathbf{0}
\end{gathered}
$$

where $e_{1}=\left(\begin{array}{cccc}1 & 0 & \ldots & 0\end{array}\right)^{T} \in \mathrm{R}^{l+1}$ and $e_{l+1}=$ $\left(\begin{array}{llll}0 & \ldots & 0 & 1\end{array}\right)^{T} \in \mathrm{R}^{l+1}$, then there exists a polynomial matrix $M(\theta)$ of same degree as $\hat{L}(\theta)$ such that $\hat{L}(\theta)=$

$$
\left\langle\left(\mathbf{1}_{\alpha} \otimes\left[\begin{array}{c}
\mathbf{0}_{1, l} \\
-\mathbf{1}_{l}
\end{array}\right] \otimes \mathbf{1}_{\beta}\right) M(\theta)\left(\mathbf{1}_{\alpha} \otimes\left[\begin{array}{ll}
\mathbf{1}_{l} & \mathbf{0}_{l, 1}
\end{array}\right] \otimes \mathbf{1}_{\beta}\right)\right\rangle
$$

Proof: The proof is direct application of Theorem 2.3.7 in [27]. Details are omited for space limitation reasons.

Lemma 3: Let $\alpha$ and $\beta$ two integers and the monoindeterminate matrix polynomial of order $2 m_{k}: L\left(\theta_{k}\right)=$ $\left(\mathbf{1}_{\alpha} \otimes \breve{\theta}_{k}^{m_{k} T} \otimes \mathbf{1}_{\beta}\right) \hat{L}\left(\mathbf{1}_{\alpha} \otimes \breve{\theta}_{k}^{m_{k}} \otimes \mathbf{1}_{\beta}\right)$. The polynomial $L\left(\theta_{k}\right)$ is identically zero for all $\theta_{k} \in \mathrm{R}$, if and only if there exists a skew-symmetric matrix $M_{k}$ such that

$$
\hat{L}=\left\langle\left(\mathbf{1}_{\alpha} \otimes \eta_{k}^{[0]} \otimes \mathbf{1}_{\beta}\right) M_{k}\left(\mathbf{1}_{\alpha} \otimes \eta_{k}^{[\infty] T} \otimes \mathbf{1}_{\beta}\right)\right\rangle .
$$

Proof: The 'if' part of the proof is omitted because similar to that of Theorem 2. The 'only if' part is now produced. For the time of the proof define the following matrices

$\Upsilon_{l}^{[0]}=\mathbf{1}_{\alpha} \otimes\left[\begin{array}{c}\mathbf{0}_{1, l} \\ -\mathbf{1}_{l}\end{array}\right] \otimes \mathbf{1}_{\beta} \quad, \quad \Upsilon_{l}^{[\infty]}=\mathbf{1}_{\alpha} \otimes\left[\begin{array}{c}\mathbf{1}_{l} \\ \mathbf{0}_{l, 1}\end{array}\right] \otimes \mathbf{1}_{\beta}$

which coincide with $\mathbf{1}_{\alpha} \otimes \eta_{k}^{[0]} \otimes \mathbf{1}_{\beta}$ and $\mathbf{1}_{\alpha} \otimes \eta_{k}^{[\infty]} \otimes \mathbf{1}_{\beta}$ respectively when $l=m_{k}$.

The lowest degree and the highest degree terms of $L$ should be zeros which writes

$$
\begin{gathered}
\left(\mathbf{1}_{\alpha} \otimes e_{1}^{T} \otimes \mathbf{1}_{\beta}\right) \hat{L}\left(\mathbf{1}_{\alpha} \otimes e_{1} \otimes \mathbf{1}_{\beta}\right)=\mathbf{0} \\
\left(\mathbf{1}_{\alpha} \otimes e_{m_{k}+1}^{T} \otimes \mathbf{1}_{\beta}\right) \hat{L}\left(\mathbf{1}_{\alpha} \otimes e_{m_{k}+1} \otimes \mathbf{1}_{\beta}\right)=\mathbf{0}
\end{gathered}
$$

which due to Lemma 2 implies the existence of a matrix $M$ such that $\hat{L}=\left\langle\Upsilon_{m_{k}}^{[0]} M \Upsilon_{m_{k}}^{[\infty] T}\right\rangle$. Decompose $M=$ $M^{\text {skew }}+M^{\text {sym }}$ in its skew-symmetric and symmetric parts. The structures of $\Upsilon_{m_{k}}^{[0]}$ and $\Upsilon_{m_{k}}^{[\infty]}$ are such that

$$
\begin{gathered}
\left(\mathbf{1}_{\alpha} \otimes \breve{\theta}_{k}^{m_{k} T} \otimes \mathbf{1}_{\beta}\right) \Upsilon_{m_{k}}^{[0]}=-\theta_{k}\left(\mathbf{1}_{\alpha} \otimes \breve{\theta}_{k}^{m_{k}-1 T} \otimes \mathbf{1}_{\beta}\right) \\
\Upsilon_{m_{k}}^{[\infty] T}\left(\mathbf{1}_{\alpha} \otimes \breve{\theta}_{k}^{m_{k}} \otimes \mathbf{1}_{\beta}\right)=\mathbf{1}_{\alpha} \otimes \breve{\theta}_{k}^{m_{k}-1} \otimes \mathbf{1}_{\beta}
\end{gathered}
$$

therefore, the polynomial $L$ being identically zero implies

$$
\begin{aligned}
\mathbf{0} & =\left(\mathbf{1}_{\alpha} \otimes \breve{\theta}_{k}^{m_{k} T} \otimes \mathbf{1}_{\beta}\right) \hat{L}\left(\mathbf{1}_{\alpha} \otimes \breve{\theta}_{k}^{m_{k}} \otimes \mathbf{1}_{\beta}\right) \\
& =-2 \theta_{k} \underbrace{\left(\mathbf{1}_{\alpha} \otimes \breve{\theta}_{k}^{m_{k}-1 T} \otimes \mathbf{1}_{\beta}\right) M^{s y m}\left(\mathbf{1}_{\alpha} \otimes \breve{\theta}_{k}^{m_{k}-1} \otimes \mathbf{1}_{\beta}\right)}_{N\left(\theta_{k}\right)}
\end{aligned}
$$


for all $\theta_{k} \in \mathrm{R}$, i.e. the polynomial $N\left(\theta_{k}\right)$ is identically zero. The remaining of the proof is done by induction on $m_{k}$ the maximal degree in the vector $\breve{\theta}_{k}^{m_{k}}=\left(\begin{array}{cccc}1 & \theta_{k} & \ldots & \theta_{k}^{m_{k}}\end{array}\right)^{T}$.

- Start with $m_{k}=1$. Then $\breve{\theta}_{k}^{m_{k}-1}=1$ and $N\left(\theta_{k}\right)=M^{\text {sym }}=\mathbf{0}$. Hence it is proved that $\hat{L}=$ $\left\langle\Upsilon_{1}^{[0]} M_{k} \Psi_{1}^{[\infty] T}\right\rangle$ with $M_{k}=M^{\text {skew }}$ skew-symmetric.

- Assume Lemma 3 holds for polynomials of order $2\left(m_{k}-1\right)$ and apply it to the polynomial $N$. It implies existence of a skew-symmetric matrix $P$ such that $M^{\text {sym }}=\left\langle\Upsilon_{m_{k}-1}^{[0]} P \Upsilon_{m_{k}-1}^{[\infty] T}\right\rangle$. At this stage of the proof one gets that $\hat{L}=\left\langle\Upsilon_{m_{k}}^{[0]} M^{\text {skew }} \Upsilon_{m_{k}}^{[\infty] T}\right\rangle+$ $\left\langle\Upsilon_{m_{k}}^{[0]}\left\langle\Upsilon_{m_{k}-1}^{[0]} P \Upsilon_{m_{k}-1}^{[\infty] T}\right\rangle \Upsilon_{m_{k}}^{[\infty] T}\right\rangle$ with $M^{\text {skew }}$ and $P$ skew-symmetric. Simple manipulations show that $\Upsilon_{m_{k}}^{[0]} \Upsilon_{m_{k}-1}^{[\infty]}=\Upsilon_{m_{k}}^{[\infty]} \Upsilon_{m_{k}-1}^{[0]}$. Therefore one gets after developping the expression and re-facorizing it properly that $\hat{L}=\left\langle\Upsilon_{m_{k}}^{[0]} M_{k} \Upsilon_{m_{k}}^{[\infty] T}\right\rangle$ where $M_{k}=$ $M^{\text {skew }}+\Upsilon_{m_{k}-1}^{[0]} P \Upsilon_{m_{k}-1}^{[\infty] T}+\Upsilon_{m_{k}-1}^{[\infty]} P \Upsilon_{m_{k}-1}^{[0] T}$ which is such that $\left\langle M_{k}\right\rangle=\left\langle M^{\text {skew }}\right\rangle+\Upsilon_{m_{k}-1}^{[0]}\langle P\rangle \Upsilon_{m_{k}-1}^{[\infty] T}+$ $\Upsilon_{m_{k}-1}^{[\infty]}\langle P\rangle \Upsilon_{m_{k}-1}^{[0] T}$. Since both matrices $M^{\text {skew }}$ and $P$ are skew-symmetric $\left(\left\langle M^{\text {skew }}\right\rangle=\mathbf{0}\right.$ and $\left.\langle P\rangle=\mathbf{0}\right), M_{k}$ is proved to be skew-symmetric $\left(\left\langle M_{k}\right\rangle=\mathbf{0}\right)$

Due to Lemma 2, if $\hat{L}$ is a polynomial matrix of some other indeterminates, $M$ may be chosen to be a polynomial with same degree with respect to these indeterminates. This fact is used in the following.

Proof of Lemma 1: Demonstration is done by induction on the number of indeterminates.

- For $\bar{k}=1$, Lemma 1 holds due to Lemma 3.

- Assume Lemma 1 holds for polynomials with $\bar{k}-1$ indeterminates and consider a polynomial $L$ of $\bar{k}$ indeterminates assumed to be identically zero. Perform the following factorization

$$
\begin{aligned}
L(\theta) & =\left(\mathbf{1}_{\alpha} \otimes \breve{\theta}_{[1, \bar{k}]}^{T} \otimes \mathbf{1}_{\beta}\right) \hat{L}\left(\mathbf{1}_{\alpha} \otimes \breve{\theta}_{[1, \bar{k}]}^{T} \otimes \mathbf{1}_{\beta}\right) \\
& =\left(\mathbf{1}_{\alpha} \otimes \breve{\theta}_{1}^{m} T \otimes \mathbf{1}_{\beta}\right) L_{1}\left(\theta_{[2, \bar{k}]}\right)\left(\mathbf{1}_{\alpha} \otimes \breve{\theta}_{1}^{m_{k}} \otimes \mathbf{1}_{\beta}\right)
\end{aligned}
$$

where $L_{1}$ is the following polynomial of $\bar{k}-1$ indeterminates $L_{1}\left(\theta_{[2, \bar{k}]}\right)=$

$$
\left(\mathbf{1}_{\alpha \sigma_{1,1}} \otimes \breve{\theta}_{[2, \bar{k}]}^{T} \otimes \mathbf{1}_{\beta}\right) \hat{L}\left(\mathbf{1}_{\alpha \sigma_{1,1}} \otimes \breve{\theta}_{[2, \bar{k}]} \otimes \mathbf{1}_{\beta}\right) .
$$

Apply Lemma 3 to the polynomial $L$ viewed as a polynomial of the first indeterminate, it implies existence of a skew-symmetric polynomial matrix $M_{1}$ of order $2 m_{k}$ with respect to each other indeterminates $\theta_{k}(k=2 \ldots \bar{k})$ such that $L_{1}\left(\theta_{[2, \bar{k}]}\right)=$

$$
\left\langle\left(\mathbf{1}_{\alpha} \otimes \eta_{1}^{[0]} \otimes \mathbf{1}_{\beta}\right) M_{1}\left(\theta_{[2, \bar{k}]}\right)\left(\mathbf{1}_{\alpha} \otimes \eta_{1}^{[\infty] T} \otimes \mathbf{1}_{\beta}\right)\right\rangle .
$$

The polynomial $M_{1}$ admits a representation in the basis of monomials as $M_{1}\left(\theta_{[2, \bar{k}]}\right)=$

$$
\left(\mathbf{1}_{\alpha m_{1}} \otimes \breve{\theta}_{[2, \bar{k}]}^{T} \otimes \mathbf{1}_{\beta}\right) \hat{M}_{1}\left(\mathbf{1}_{\alpha m_{1}} \otimes \breve{\theta}_{[2, \bar{k}]} \otimes \mathbf{1}_{\beta}\right) .
$$

Decompose $\hat{M}_{1}=\hat{M}_{1}^{\text {skew }}+\hat{M}_{1}^{\text {sym }}$ in its skew-symmetric and symmetric parts. The constraint that $M_{1}\left(\theta_{[2, \bar{k}]}\right)$ is skew-symmetric implies

$\mathbf{0}=2\left(\mathbf{1}_{\alpha m_{1}} \otimes \breve{\theta}_{[2, \bar{k}]}^{T} \otimes \mathbf{1}_{\beta}\right) \hat{M}_{1}^{s y m}\left(\mathbf{1}_{\alpha m_{1}} \otimes \breve{\theta}_{[2, \bar{k}]} \otimes \mathbf{1}_{\beta}\right)$.

$\hat{M}_{1}^{\text {sym }}$ therefore defines a polynomial of $\bar{k}-1$ indeterminates which is identically zero. Lemma 1 is assumed to hold for polynomials of $\bar{k}-1$ indeterminates, thus

$$
\begin{array}{r}
\hat{M}_{1}^{\text {sym }}=\left\langle\sum_{k=2}^{\bar{k}}\left(\mathbf{1}_{\alpha m_{1} \sigma_{2, k-1}} \otimes \eta_{k}^{[0]} \otimes \mathbf{1}_{\beta \sigma_{k+1, \bar{k}}}\right) \hat{N}_{1 k} \ldots\right. \\
\left.\left(\mathbf{1}_{\alpha m_{1} \sigma_{2, k-1}} \otimes \eta_{k}^{[\infty] T} \otimes \mathbf{1}_{\beta \sigma_{k+1, \bar{k}}}\right)\right\rangle
\end{array}
$$

with skew-symmetric matrices $\hat{N}_{1 k}$. Simple manipulations show that whatever $k=2 \ldots \bar{k}$ and whatever $(i, j) \in\{0, \infty\}^{2}$ the following formula hold

$$
\begin{aligned}
& \left(\mathbf{1}_{\alpha} \otimes \eta_{1}^{[i]} \otimes \mathbf{1}_{\beta}\right)\left(\mathbf{1}_{\alpha m_{1} \sigma_{2, k-1}} \otimes \eta_{k}^{[j]} \otimes \mathbf{1}_{\beta \sigma_{k+1, \bar{k}}}\right) \\
& =\left(\mathbf{1}_{\alpha \sigma_{1, k-1}} \otimes \eta_{k}^{[j]} \otimes \mathbf{1}_{\beta \sigma_{k+1, \bar{k}}}\right)\left(\mathbf{1}_{\alpha} \otimes \eta_{1}^{[i]} \otimes \mathbf{1}_{\tilde{\beta}_{k}}\right)
\end{aligned}
$$

where $\tilde{\beta}_{k}=\beta \sigma_{2, \bar{k}} m_{k} /\left(m_{k}+1\right)$. Therefore with a quite similar operations as in the proof of Lemma 3, one gets that

$$
\begin{array}{r}
\left\langle\left(\mathbf{1}_{\alpha} \otimes \eta_{1}^{[0]} \otimes \mathbf{1}_{\beta}\right) \hat{M}_{1}^{s y m}\left(\mathbf{1}_{\alpha} \otimes \eta_{1}^{[\infty] T} \otimes \mathbf{1}_{\beta}\right)\right\rangle \\
=\left\langle\sum_{k=2}^{\bar{k}}\left(\mathbf{1}_{\alpha \sigma_{1, k-1}} \otimes \eta_{k}^{[0]} \otimes \mathbf{1}_{\beta \sigma_{k+1, \bar{k}}}\right) \tilde{N}_{k} \ldots\right. \\
\left.\left(\mathbf{1}_{\alpha \sigma_{1, k-1}} \otimes \eta_{k}^{[\infty] T} \otimes \mathbf{1}_{\beta \sigma_{k+1, \bar{k}}}\right)\right\rangle
\end{array}
$$

where $\tilde{N}_{k}=\left(\mathbf{1}_{\alpha} \otimes \eta_{1}^{[0]} \otimes \mathbf{1}_{\tilde{\beta}_{k}}\right) \hat{N}_{1, k}\left(\mathbf{1}_{\alpha} \otimes \eta_{1}^{[\infty] T} \otimes\right.$ $\left.\mathbf{1}_{\tilde{\beta}_{k}}\right)+\left(\mathbf{1}_{\alpha} \otimes \eta_{1}^{[\infty]} \otimes \mathbf{1}_{\tilde{\beta}_{k}}\right) \hat{N}_{1, k}\left(\mathbf{1}_{\alpha} \otimes \eta_{1}^{[0] T} \otimes \mathbf{1}_{\tilde{\beta}_{k}}\right)$. The matrices $\tilde{N}_{k}$ happen to be skew-symmetric because $\hat{N}_{1, k}$ are skew-symmetric. Denote $\tilde{N}_{1}=\hat{M}_{1}^{\text {skew }}$. At this stage, combining equations (14), (15) and (16) gives

$L_{1}\left(\theta_{[2, \bar{k}]}\right)=\left(\mathbf{1}_{\alpha \sigma_{1,1}} \otimes \breve{\theta}_{[2, \bar{k}]}^{T} \otimes \mathbf{1}_{\beta}\right) \hat{L}_{1}\left(\mathbf{1}_{\alpha \sigma_{1,1}} \otimes \breve{\theta}_{[2, \bar{k}]} \otimes \mathbf{1}_{\beta}\right)$

where $\hat{L}_{1}=$

$$
\begin{array}{r}
\left\langle\sum_{k=1}^{\bar{k}}\left(\mathbf{1}_{\alpha \sigma_{1, k-1}} \otimes \eta_{k}^{[0]} \otimes \mathbf{1}_{\beta \sigma_{k+1, \bar{k}}}\right) \tilde{N}_{k} \ldots\right. \\
\left.\left(\mathbf{1}_{\alpha \sigma_{1, k-1}} \otimes \eta_{k}^{[\infty] T} \otimes \mathbf{1}_{\beta \sigma_{k+1, \bar{k}}}\right)\right\rangle
\end{array}
$$

with all matrices $\tilde{N}_{k}$ being skew-symmetric. Subtracting expression (17) to (13) gives

$$
\left(\mathbf{1}_{\alpha \sigma_{1,1}} \otimes \breve{\theta}_{[2, \bar{k}]}^{T} \otimes \mathbf{1}_{\beta}\right)\left(\hat{L}-\hat{L}_{1}\right)\left(\mathbf{1}_{\alpha \sigma_{1,1}} \otimes \breve{\theta}_{[2, \bar{k}]} \otimes \mathbf{1}_{\beta}\right)=\mathbf{0}
$$

for all $\theta_{[2, \bar{k}]} \in \mathrm{R}^{\bar{k}-1}$. This new polynomial matrix of $\bar{k}-1$ indeterminates is identically zero. Lemma 3 is assumed to hold for such polynomials of $\bar{k}-1$ indeterminates, therefore there exist $\bar{k}-1$ skew-symmetric matrices $\hat{N}_{2, k}$ $(k=2 \ldots \bar{k})$ such that $\hat{L}-\hat{L}_{1}=$

$$
\begin{array}{r}
\left\langle\sum_{k=2}^{\bar{k}}\left(\mathbf{1}_{\alpha \sigma_{1, k-1}} \otimes \eta_{k}^{[0]} \otimes \mathbf{1}_{\beta \sigma_{k+1, \bar{k}}}\right) \hat{N}_{2, k} \ldots\right. \\
\left.\left(\mathbf{1}_{\alpha \sigma_{1, k-1}} \otimes \eta_{k}^{[\infty] T} \otimes \mathbf{1}_{\beta \sigma_{k+1, \bar{k}}}\right)\right\rangle .
\end{array}
$$

Combining (18) and (19) proves the result where $\hat{N}_{1}=$ $\tilde{N}_{1}=\hat{M}_{1}^{\text {skew }}$ and $\hat{N}_{k}=\hat{N}_{2, k}+\tilde{N}_{k}$ for $k=2 \ldots \bar{k}$. 